Entecavir is an anti-viral drug commonly used in treatment of hepatitis-B infection but there was no recognised ocular toxicity in its association [1-3]. The mechanism of retinal toxicity remains unclear with lacking histopathological studies. There was a published case report with irreversible blindness when on year(s) of Entecavir complicated by diabetic retinopathy at the time of suspected retinal toxicity [4]. Our case shared similar clinical features as their reported second eye. Notably, Entecavir drug toxicity is causing sequential rather than bilateral simultaneous eye manifestations. Our patient's left eye was profoundly involved but his right eye had not progressed more than the minimal peripheral vascular staining in late fundus photographs and fluorescein angiography phase (Fig. 1: 2A).

Our case confirmed short-term Entecavir use could cause reversible retinal toxicity upon drug cessation. Prescribing physicians and ophthalmologists should be aware of the potential retinal toxicity of Entecavir. We reported this adverse drug reaction to the UK Medicines and Health Products Regulatory Agency.
Acknowledgements We thanked Dr. Peter Good/Dr. Emma Berrow with their visual function team and Ms. Rosie Auld with her orthoptic team for their accommodative prompt services in performing/interpreting the results of visual function tests at various stages.

\section{Compliance with ethical standards}

Conflict of interest The authors declare that they have no conflict of interest.

\section{References}

1. Entecavir. National Center for Biotechnology Information. Pubchem compound database; $\mathrm{CID}=153941$. http://pubchem.ncbi.nlm. nih.gov/compound/153941.

2. Wong GL, Seto WK, Wong VW, Yuen MF, Chan HL. Review article: long-term safety of oral anti-viral treatment for chronic hepatitis-B. Aliment Pharmacol Ther. 2018;47:730-7.

3. Kayaaslan B, Guner R. Adverse effects of oral antiviral therapy in chronic hepatitis B. World J Hepatol. 2017;9:227-41.

4. Muqit MK, Stanga PE, Vilar FJ, Patton N. Presumed entecavirinduced ocular toxicity. Eye. 2011;25:1665-8.

\title{
Christmas tree cataract and myotonic dystrophy type 1
}

\author{
Dionysios Pagoulatos ${ }^{1} \cdot$ Zoi Kapsala $^{2} \cdot$ Olga E. Makri $^{1} \cdot$ Constantinos D. Georgakopoulos $^{1}$
}

Received: 14 March 2018 / Revised: 2 May 2018 / Accepted: 1 June 2018 / Published online: 9 July 2018

(c) The Royal College of Ophthalmologists 2018

We have read with great interest the recent article by Papadopoulos et al. [1] reporting early onset posterior subscapular cataract in 9 out of 28 patients with myotonic dystrophy type 2 (DM2). They also mentioned that this type of cataract was the first symptom in $7(25 \%)$ of the studied DM2 patients.

Dionysios Pagoulatos pagdion@gmail.com

1 Department of Ophthalmology, School of Medicine, University of Patras, Patras, Greece

2 Department of Ophthalmology, School of Medicine, University of Crete, Heraklion, Greece
Here, we would like to present our results regarding the incidence of Christmas tree cataract in patients with the most common form of DM, myotonic dystrophy type 1 (DM1). Christmas tree cataract is considered a characteristic finding in subjects with DM1. The retrospective review of medical records of 23 patients with DM1 revealed the presence of Christmas tree cataract in 13 patients $(56 \%)$. The multicoloured, iridescent lens opacities were unilateral in 10 out of the 13 patients and asymmetric bilateral in 3 patients. Age when cataract was diagnosed was $47 \pm 5$ years (range: $35-52$ years). The cataract was the first sign of the disease for 11 patients and was detected accidentally during a routine ophthalmological examination. Best corrected visual acuity was $0.06 \pm 0.08 \log$ MAR (range: -0.1 to $0.2 \operatorname{logMAR}$ ). The interval between diagnosis of cataract and DM1 was $10 \pm 2$ years (range: 
7-14 years). This delay is explained by the fact that none of the patients presented typical symptoms of DM1 at the time of the diagnosis of the cataract. Remarkably, apart from Christmas tree cataract, no other form of cataract was detected in the study group, as well as no other pathology regarding the anterior or posterior segment was recorded.

Notably, in previous studies, Christmas tree cataract has been detected in nearly all patients with DM1, and viceversa $16.7 \%$ of subjects with Christmas tree cataract have been diagnosed with DM1 [2].

In conclusion, earlier detection of Christmas tree cataract, which constitutes a common ophthalmologic finding in DM1 patients [3, 4], and thus, referral for neuromuscular assessment could possibly eliminate delays in diagnosis of the disease. Noteworthy, it seems that Christmas cataract may be the first manifestation of DM1 in a higher percentage of patients than posterior subcapsular cataract in DM2 patients. Extended multicenter studies may elucidate the exact incidence of the different cataract types in these patients.

\section{Compliance with ethical standards}

Conflict of interest The authors declare that they have no conflict of interest.

Informed consent Written informed consent was obtained from all patients and the study was approved by the institutional review board at the University of Patras.

\section{References}

1. Papadopoulos C, Kekou K, Xirou S, Kitsiou-Tzeli S, Kararizou E, Papadimas GK. Early onset posterior subscapular cataract in a series of myotonic dystrophy type 2 patients. Eye (Lond). 2018;32:622-625

2. Reiter C, Gramer E. Anticipation in patients with iridescent multicoloured posterior capsular lens opacities ("Christmas tree cataract"): the role in the diagnosis of myotonic dystrophy. Ophthalmologe. 2009;106:1116-20.

3. Bird TD. Myotonic dystrophy type 1. In: Adam MP, Ardinger HH, Pagon RA, et al., editors. GeneReview [Internet]. Seattle, WA: University of Washington; Seattle; 1999. p. 1993-2017.

4. Stival L, Bittar R, Lago A, Nassaralla J Jr. Christmas tree cataract. Rev Bras Oftalmol. 2015;74:309-11.

\title{
Hypothyroidism and non-infectious uveitis
}

\author{
Shorya Vardhan Azad ${ }^{1} \cdot$ Varun Gogia $^{1} \cdot$ Pradeep Venkatesh $^{1} \cdot$ Brijesh Takkar $^{1}$
}

Received: 7 February 2018 / Accepted: 12 February 2018 / Published online: 24 July 2018

(c) The Royal College of Ophthalmologists 2018

Understanding the non-specific symptoms of uveitis and its corroboration with systemic findings may give insight into its pathophysiology and may in turn help in its management. Association of thyroid disorders with uveitis is well known; however, emphasis has not been made in most of the past studies regarding its prevalence and spectrum of presentation $[1,2]$.

We performed a prospective analysis of patients presenting at our uvea clinic at a tertiary eye care center in Northern India and evaluated the patterns of uveitis in our

Pradeep Venkatesh

venkyprao@yahoo.com

1 Dr. Rajendra Prasad Centre for Ophthalmic Sciences, AIIMS, New Delhi, India population. As a part of study we also analyzed the prevalence of associated systemic diseases and systemic symptoms. We observed that $24.5 \%$ of patients of uveitis had underlying systemic disease, where past or present evidence of tuberculosis was most common followed by seronegative spondyloarthropathy and sarcoidosis with overall prevalence of $6.8 \%, 4.1 \%$, and $2.5 \%$, respectively. Prevalence of hypothyroidism in these patients was $1.5 \%$ amongst (12 patients out of 800 ) all patients and 6.1\% among patients with known systemic illness. There were 10 females and 2 males with median age of 37 years (Table 1). Spectrum of involvement was varied, with intermediate uveitis (IU) being the most common presentation. All patients had confirmed hypothyroidism and were on thyroid replacement therapy. None of them suffered from hyperthyroidism. Thyroid functions tests and anti-thyroid 